\title{
Digital language of Atomic Weights of the Chemical Elements
}

\author{
Lutvo Kurić \\ Institute of Economics, University of Sarajevo, Trg Oslobođenja 1, Sarajevo, \\ Bosnia and Herzegovina \\ E-mail address: lutvokuric@yahoo.com
}

\begin{abstract}
The subject of this thesis is a digital approach to the investigation of the digital basis of digital Periodic Table - periods 4 and 5. A period 4 element is one of the chemical elements in the fourth row (or period) of the periodic table of the elements. The fourth period contains 18 elements, beginning with potassium and ending with krypton. As a rule, period 4 elements fill their $4 \mathrm{~s}$ shells first, then their $3 \mathrm{~d}$ and $4 \mathrm{p}$ shells, in that order, however there are exceptions, such as chromium. A period 5 element is one of the chemical elements in the fifth row (or period) of the periodic table of the elements. The fifth period contains 18 elements, beginning with rubidium and ending with xenon. As a rule, period 5 elements fill their $5 \mathrm{~s}$ shells first, then their $4 \mathrm{~d}$, and $5 \mathrm{p}$ shells, in that order, however there are exceptions, such as rhodium. The digital mechanism of those periods have been analyzed by the application of cybernetic methods, information theory and system theory, respectively. This paper is to report that we discovered new methods for development of the new technologies in chemistry. It is about the most advanced digital technology which is based on program, cybernetics and informational systems and laws. The results in practical application of the new technology could be useful in chemistry, bioinformatics, genetics, bio-chemistry and other natural sciences.
\end{abstract}

Keywords: Digital Periodic Table; period 4 element; period 5 element; digital chemistry; digital chemical code

\section{INTRODUCTION}

The subjects of our research are program lawfulness, cybernetic lawfulness, and informational lawfulness in Periodic system Table, fourth and fifth periods. In the science, one question has been present for a long time, that is, if there is one unique common connection that links all chemical elements in this Table. The doubt is, if the periodical is only a physical-chemical matter of objective material relationship or maybe a matter of numbers and mathematics. With the goal to find the answers on some of those questions, we have made a decision to do a research on, if in this Table exists program, cybernetic and information lawfulness. Results is: We have discovered that sequences of all elements in this Table conducted, not just according to their chemical and periodical characteristics, but especially according to the program lawfulness, cybernetic lawfulness, and informational lawfulness. In fact, we have discovered the digital balance in distribution of elements in Periodic system Table is achieved. Here we wish to present our points of views about the program-cybernetics lawfulness in this Table. 


\section{RESULTS}

Results of our research show that the processes of sequencing the chemical elements in the fourth and fifth periods are conditioned and arranged not only with chemical and biochemical, but also with program, cybernetic and informational lawfulness too. At the first stage of our research we replaced chemical elements from the Periodic Table with atomic numbers of those elements. This study translates the periodic table of elements from a digital form and explores the idea of improving readers' comprehension and retention of complex information. It is designed to help readers visualize abstract information by actively engaging them in their learning experience. It also helps them understand the interconnectedness of complex systems the periodic table of elements being a prime example by translating digital numerical information into visual patterns that can be detected and compared. Users will hopefully apply this form of learning to other areas as well.

\section{Decode the digital chemical language}

The above algorithms enable to decode the digital chemical language and to discover codes that mutually connect the parameters in digital images from Periodic Table - periods 4 and 5.

\section{Examples:}

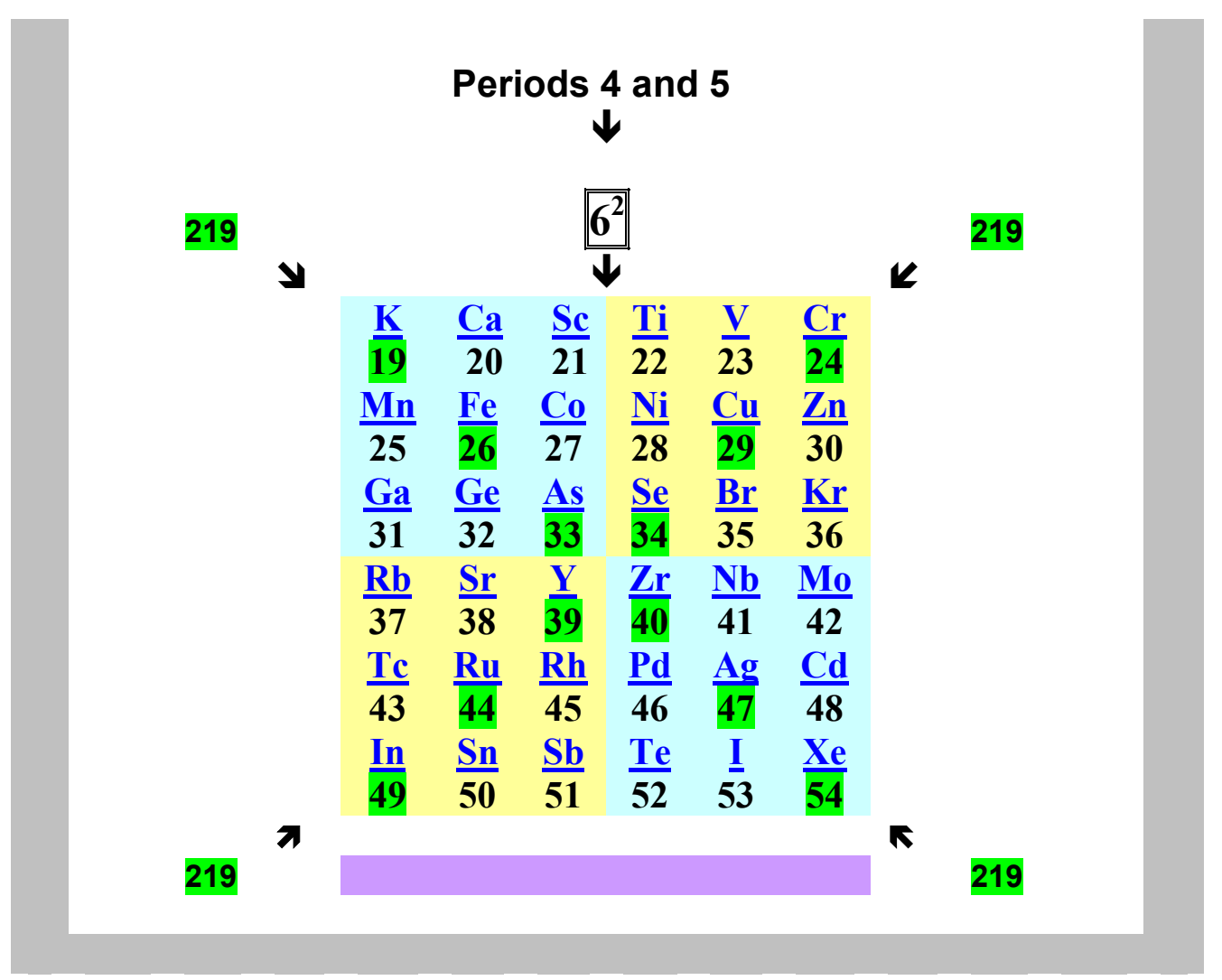


Digital fourth and fifth periods

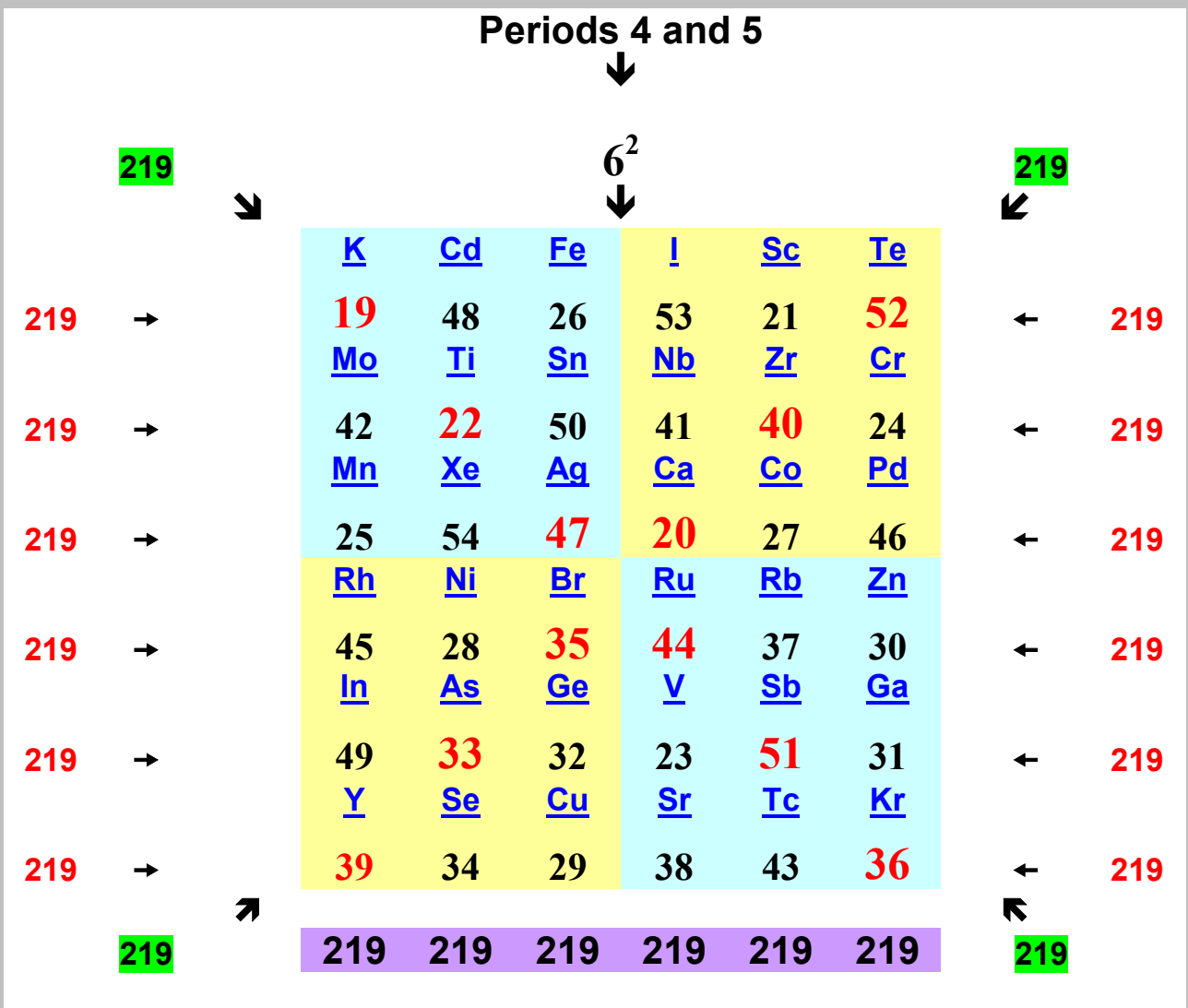

ToeplitzMatrix $[(19,48,26,53,21,52,42,22,50,41,40,24,25,54,47,20,27,46)]$

Input:

ToeplitzMatrix $[(19,48,26,53,21,52,42,22,50,41,40,24,25,54,47,20,27,46)]$ 


\section{Result:}

$\left(\begin{array}{llllllllllllllllll}19 & 48 & 26 & 53 & 21 & 52 & 42 & 22 & 50 & 41 & 40 & 24 & 25 & 54 & 47 & 20 & 27 & 46 \\ 48 & 19 & 48 & 26 & 53 & 21 & 52 & 42 & 22 & 50 & 41 & 40 & 24 & 25 & 54 & 47 & 20 & 27 \\ 26 & 48 & 19 & 48 & 26 & 53 & 21 & 52 & 42 & 22 & 50 & 41 & 40 & 24 & 25 & 54 & 47 & 20 \\ 53 & 26 & 48 & 19 & 48 & 26 & 53 & 21 & 52 & 42 & 22 & 50 & 41 & 40 & 24 & 25 & 54 & 47 \\ 21 & 53 & 26 & 48 & 19 & 48 & 26 & 53 & 21 & 52 & 42 & 22 & 50 & 41 & 40 & 24 & 25 & 54 \\ 52 & 21 & 53 & 26 & 48 & 19 & 48 & 26 & 53 & 21 & 52 & 42 & 22 & 50 & 41 & 40 & 24 & 25 \\ 42 & 52 & 21 & 53 & 26 & 48 & 19 & 48 & 26 & 53 & 21 & 52 & 42 & 22 & 50 & 41 & 40 & 24 \\ 22 & 42 & 52 & 21 & 53 & 26 & 48 & 19 & 48 & 26 & 53 & 21 & 52 & 42 & 22 & 50 & 41 & 40 \\ 50 & 22 & 42 & 52 & 21 & 53 & 26 & 48 & 19 & 48 & 26 & 53 & 21 & 52 & 42 & 22 & 50 & 41 \\ 41 & 50 & 22 & 42 & 52 & 21 & 53 & 26 & 48 & 19 & 48 & 26 & 53 & 21 & 52 & 42 & 22 & 50 \\ 40 & 41 & 50 & 22 & 42 & 52 & 21 & 53 & 26 & 48 & 19 & 48 & 26 & 53 & 21 & 52 & 42 & 22 \\ 24 & 40 & 41 & 50 & 22 & 42 & 52 & 21 & 53 & 26 & 48 & 19 & 48 & 26 & 53 & 21 & 52 & 42 \\ 25 & 24 & 40 & 41 & 50 & 22 & 42 & 52 & 21 & 53 & 26 & 48 & 19 & 48 & 26 & 53 & 21 & 52 \\ 54 & 25 & 24 & 40 & 41 & 50 & 22 & 42 & 52 & 21 & 53 & 26 & 48 & 19 & 48 & 26 & 53 & 21 \\ 47 & 54 & 25 & 24 & 40 & 41 & 50 & 22 & 42 & 52 & 21 & 53 & 26 & 48 & 19 & 48 & 26 & 53 \\ 20 & 47 & 54 & 25 & 24 & 40 & 41 & 50 & 22 & 42 & 52 & 21 & 53 & 26 & 48 & 19 & 48 & 26 \\ 27 & 20 & 47 & 54 & 25 & 24 & 40 & 41 & 50 & 22 & 42 & 52 & 21 & 53 & 26 & 48 & 19 & 48 \\ 46 & 27 & 20 & 47 & 54 & 25 & 24 & 40 & 41 & 50 & 22 & 42 & 52 & 21 & 53 & 26 & 48 & 19\end{array}\right)$

Dimensions:

18 (rows) $\times 18$ (columns)

Matrix plot:

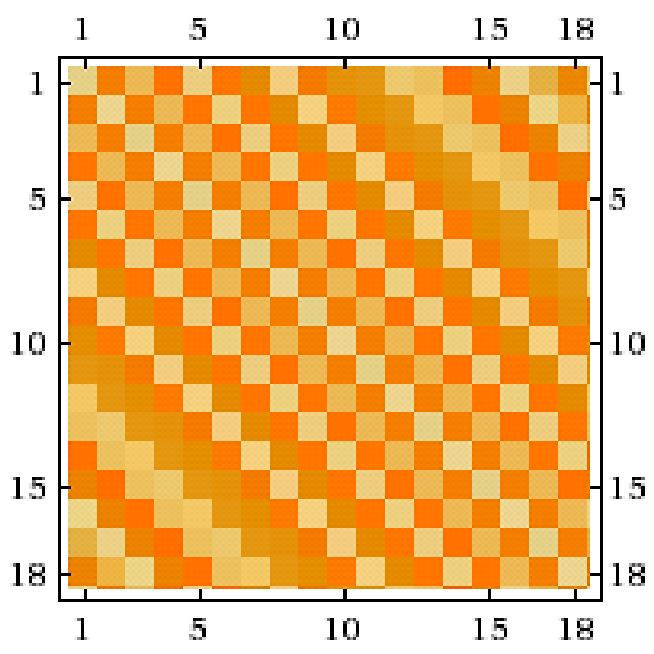

Properties:

symmetric

Determinant:

156511602959420674132878744

Trace:

342 
Characteristic polynomial:

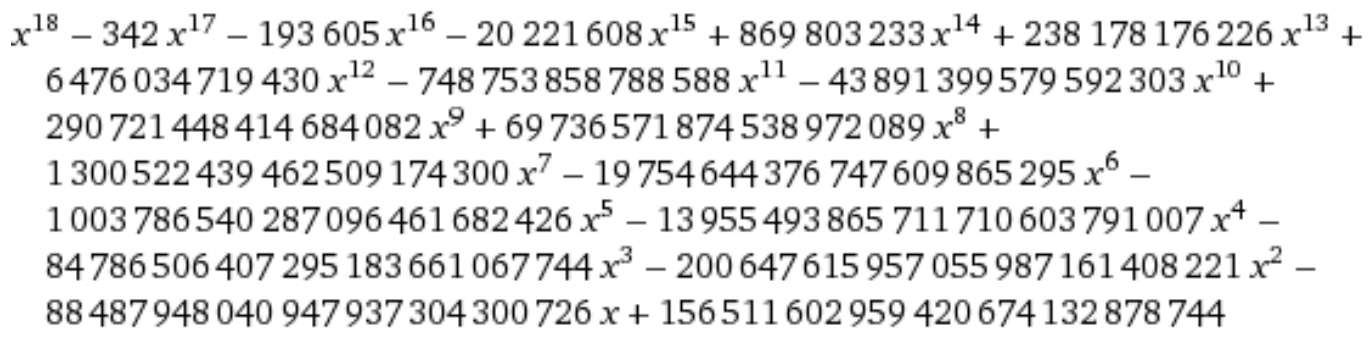

Condition number:

1841.57

\begin{tabular}{|c|c|c|c|c|c|c|c|c|c|c|c|c|c|c|c|c|}
\hline 19 & 48 & 26 & 53 & 21 & 52 & 42 & 22 & 50 & 41 & 40 & 24 & 25 & 54 & 47 & 20 & 27 \\
\hline 48 & 19 & 48 & 26 & 53 & 21 & 52 & 42 & 22 & 50 & 41 & 40 & 24 & 25 & 54 & 47 & 20 \\
\hline 26 & 48 & 19 & 48 & 26 & 53 & 21 & 52 & 42 & 22 & 50 & 41 & 40 & 24 & 25 & 54 & 47 \\
\hline 53 & 26 & 48 & 19 & 48 & 26 & 53 & 21 & 52 & 42 & 22 & 50 & 41 & 40 & 24 & 25 & 54 \\
\hline 21 & 53 & 26 & 48 & 19 & 48 & 26 & 53 & 21 & 52 & 42 & 22 & 50 & 41 & 40 & 24 & 25 \\
\hline 52 & 21 & 53 & 26 & 48 & 19 & 48 & 26 & 53 & 21 & 52 & 42 & 22 & 50 & 41 & 40 & 24 \\
\hline 42 & 52 & 21 & 53 & 26 & 48 & 19 & 48 & 26 & 53 & 21 & 52 & 42 & 22 & 50 & 41 & 40 \\
\hline 22 & 42 & 52 & 21 & 53 & 26 & 48 & 19 & 48 & 26 & 53 & 21 & 52 & 42 & 22 & 50 & 41 \\
\hline 50 & 22 & 42 & 52 & 21 & 53 & 26 & 48 & 19 & 48 & 26 & 53 & 21 & 52 & 42 & 22 & 50 \\
\hline 41 & 50 & 22 & 42 & 52 & 21 & 53 & 26 & 48 & 19 & 48 & 26 & 53 & 21 & 52 & 42 & 22 \\
\hline 40 & 41 & 50 & 22 & 42 & 52 & 21 & 53 & 26 & 48 & 19 & 48 & 26 & 53 & 21 & 52 & 42 \\
\hline 24 & 40 & 41 & 50 & 22 & 42 & 52 & 21 & 53 & 26 & 48 & 19 & 48 & 26 & 53 & 21 & 52 \\
\hline 25 & 24 & 40 & 41 & 50 & 22 & 42 & 52 & 21 & 53 & 26 & 48 & 19 & 48 & 26 & 53 & 21 \\
\hline 54 & 25 & 24 & 40 & 41 & 50 & 22 & 42 & 52 & 21 & 53 & 26 & 48 & 19 & 48 & 26 & 53 \\
\hline 47 & 54 & 25 & 24 & 40 & 41 & 50 & 22 & 42 & 52 & 21 & 53 & 26 & 48 & 19 & 48 & 26 \\
\hline 20 & 47 & 54 & 25 & 24 & 40 & 41 & 50 & 22 & 42 & 52 & 21 & 53 & 26 & 48 & 19 & 48 \\
\hline 27 & 20 & 47 & 54 & 25 & 24 & 40 & 41 & 50 & 22 & 42 & 52 & 21 & 53 & 26 & 48 & 19 \\
\hline 46 & 27 & 20 & 47 & 54 & 25 & 24 & 40 & 41 & $\mathbf{5 0}$ & 22 & 42 & 52 & 21 & 53 & 26 & 48 \\
\hline
\end{tabular}

Atomic numbers

$3005 \quad 3034$

\begin{tabular}{|c|c|c|c|c|c|c|c|c|c|c|c|c|c|c|c|c|c|c|}
\hline & & & & & & & & & & & & & & & & & & \\
\hline 19 & 48 & 26 & 53 & 21 & 52 & 42 & 22 & 50 & 41 & 40 & 24 & 25 & 54 & 47 & 20 & 27 & 46 & 657 \\
\hline 48 & 19 & 48 & 26 & 53 & 21 & 52 & 42 & 22 & 50 & 41 & 40 & 24 & 25 & 54 & 47 & 20 & 27 & 659 \\
\hline 26 & 48 & 19 & 48 & 26 & 53 & 21 & 52 & 42 & 22 & 50 & 41 & 40 & 24 & 25 & 54 & 47 & 20 & 658 \\
\hline 53 & 26 & 48 & 19 & 48 & 26 & 53 & 21 & 52 & 42 & 22 & 50 & 41 & 40 & 24 & 25 & 54 & 47 & 691 \\
\hline 21 & 53 & 26 & 48 & 19 & 48 & 26 & 53 & 21 & 52 & 42 & 22 & 50 & 41 & 40 & 24 & 25 & 54 & 665 \\
\hline 52 & 21 & 53 & 26 & 48 & 19 & 48 & 26 & 53 & 21 & 52 & 42 & 22 & 50 & 41 & 40 & 24 & 25 & 663 \\
\hline 42 & 52 & 21 & 53 & 26 & 48 & 19 & 48 & 26 & 53 & 21 & 52 & 42 & 22 & 50 & 41 & 40 & 24 & 680 \\
\hline 22 & 42 & 52 & 21 & 53 & 26 & 48 & 19 & 48 & 26 & 53 & 21 & 52 & 42 & 22 & 50 & 41 & 40 & 678 \\
\hline 50 & 22 & 42 & 52 & 21 & 53 & 26 & 48 & 19 & 48 & 26 & 53 & 21 & 52 & 42 & 22 & 50 & 41 & 688 \\
\hline 41 & 50 & 22 & 42 & 52 & 21 & 53 & 26 & 48 & 19 & 48 & 26 & 53 & 21 & 52 & 42 & 22 & 50 & 688 \\
\hline 40 & 41 & 50 & 22 & 42 & 52 & 21 & 53 & 26 & 48 & 19 & 48 & 26 & 53 & 21 & 52 & 42 & 22 & 678 \\
\hline 24 & 40 & 41 & 50 & 22 & 42 & 52 & 21 & 53 & 26 & 48 & 19 & 48 & 26 & 53 & 21 & 52 & 42 & 680 \\
\hline 25 & 24 & 40 & 41 & 50 & 22 & 42 & 52 & 21 & 53 & 26 & 48 & 19 & 48 & 26 & 53 & 21 & 52 & 663 \\
\hline 54 & 25 & 24 & 40 & 41 & 50 & 22 & 42 & 52 & 21 & 53 & 26 & 48 & 19 & 48 & 26 & 53 & 21 & 665 \\
\hline 47 & 54 & 25 & 24 & 40 & 41 & 50 & 22 & 42 & 52 & 21 & 53 & 26 & 48 & 19 & 48 & 26 & 53 & 691 \\
\hline 20 & 47 & 54 & 25 & 24 & 40 & 41 & 50 & 22 & 42 & 52 & 21 & 53 & 26 & 48 & 19 & 48 & 26 & 658 \\
\hline 27 & 20 & 47 & 54 & 25 & 24 & 40 & 41 & 50 & 22 & 42 & 52 & 21 & 53 & 26 & 48 & 19 & 48 & 659 \\
\hline 46 & 27 & 20 & 47 & 54 & 25 & 24 & 40 & 41 & 50 & 22 & 42 & 52 & 21 & 53 & 26 & 48 & 19 & 657 \\
\hline & & & 3034 & & & & & & & & & 3005 & & & & & & \\
\hline 657 & 659 & 658 & 691 & 665 & 663 & 680 & 678 & & 688 & 678 & 680 & 663 & 665 & 691 & 658 & 659 & 657 & \\
\hline
\end{tabular}




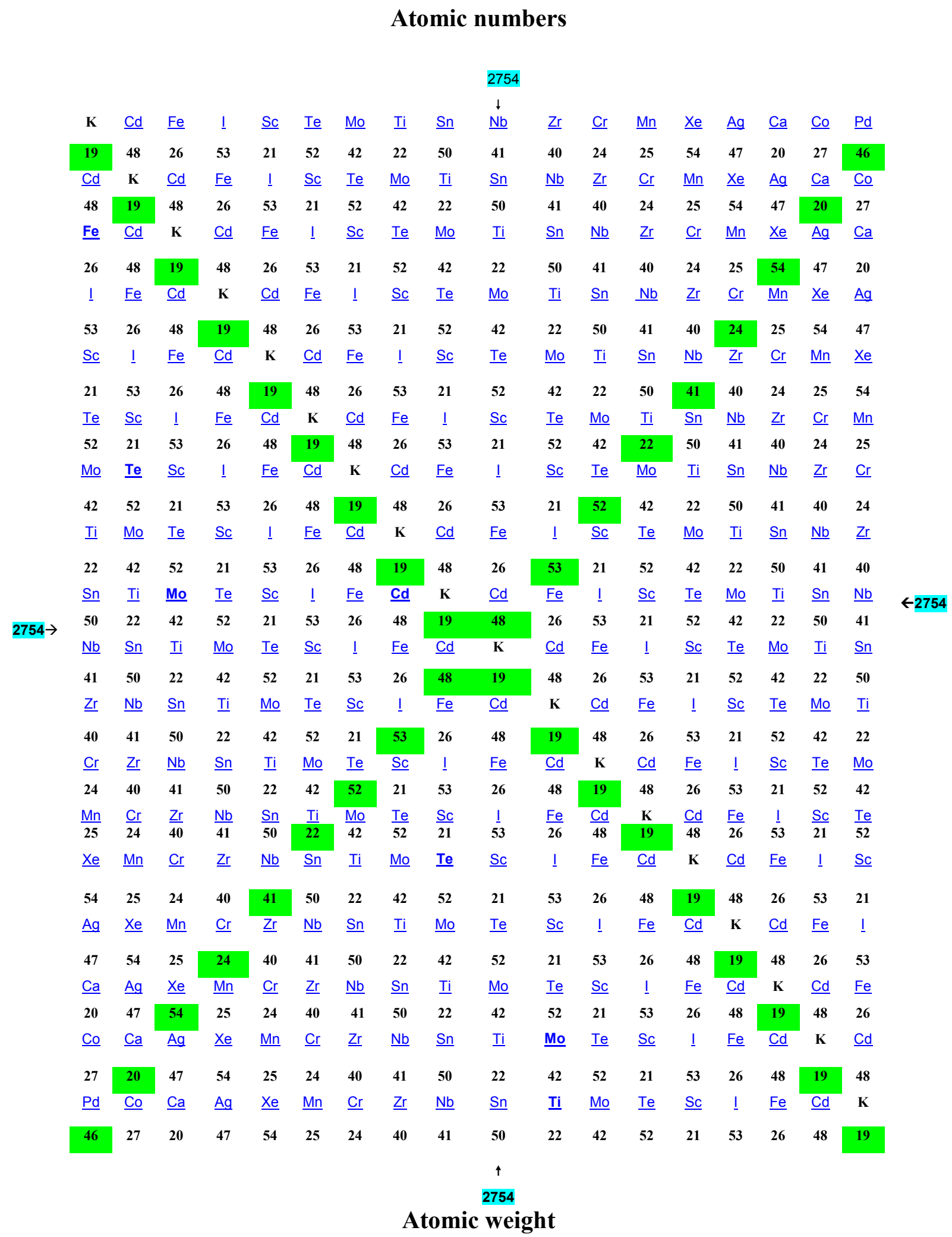

The average mass of atoms of an element, calculated using the relative abundance of isotopes in a naturally-occurring element. It is the weighted average of the masses of naturally-occurring isotopes. 


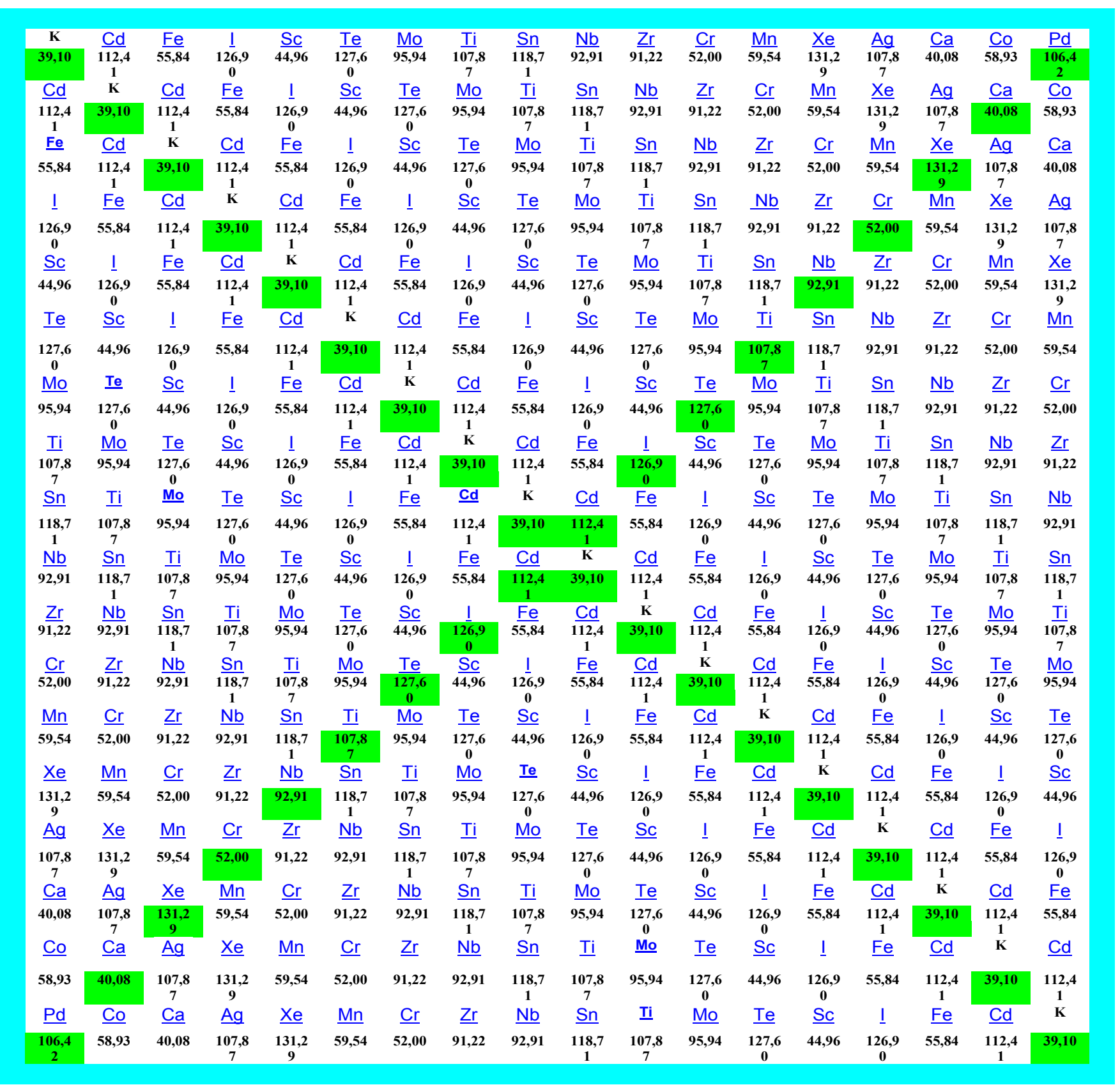




\section{Atomicweight-fragment 1 .}

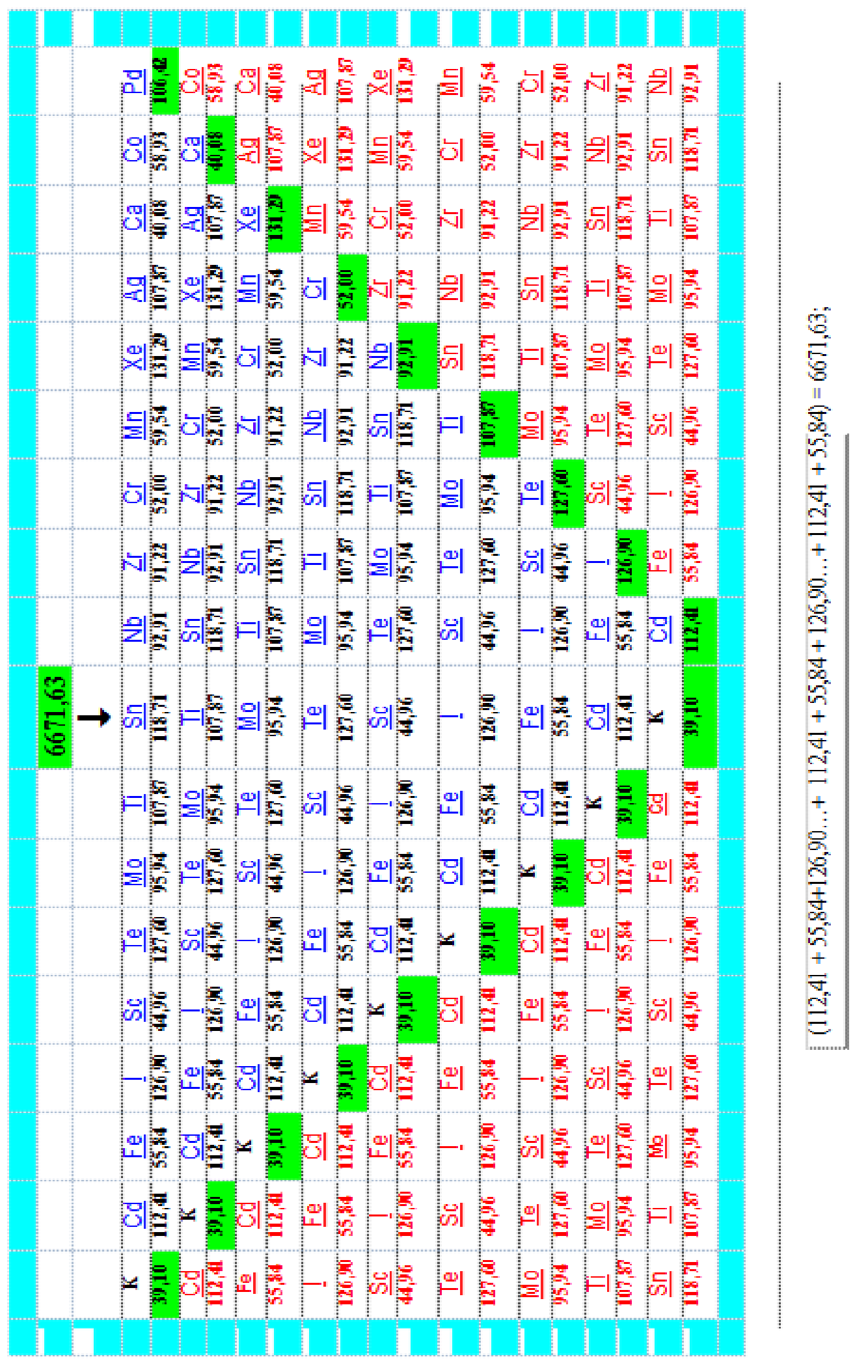


Atomic weight-fragment 2.

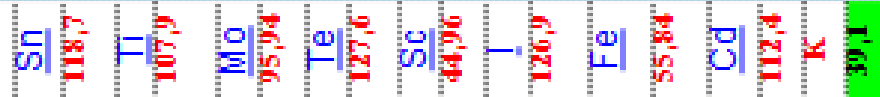

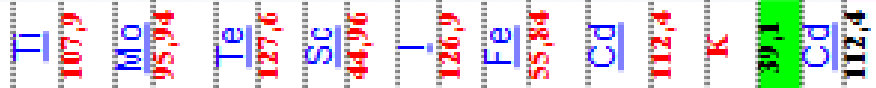

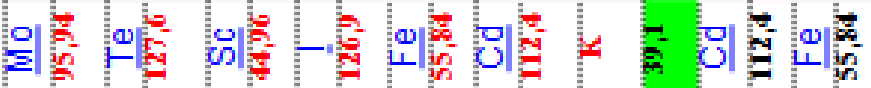

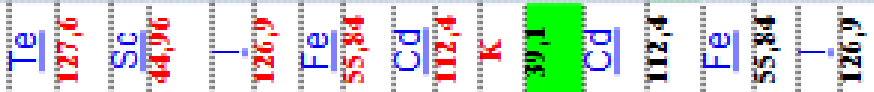

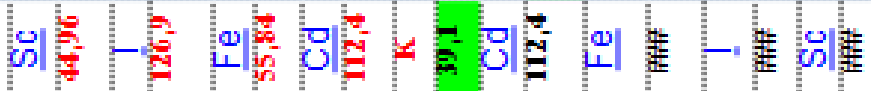

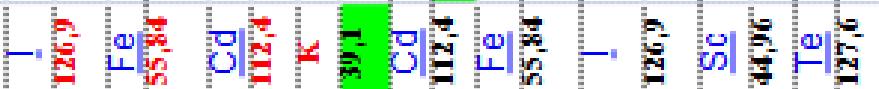

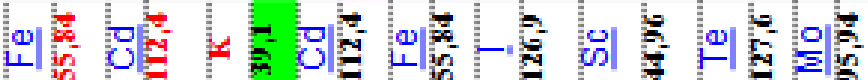

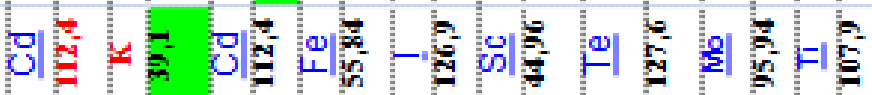

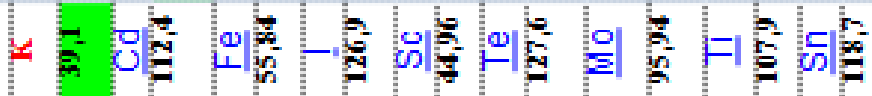

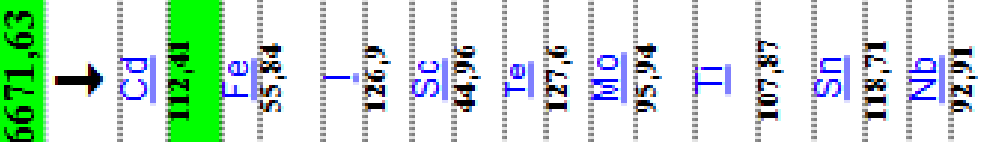

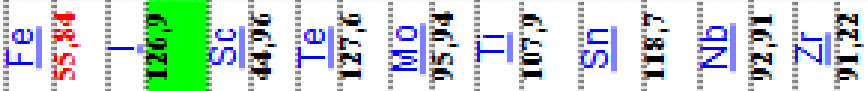

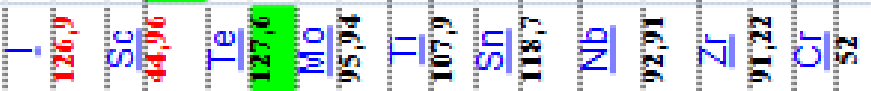

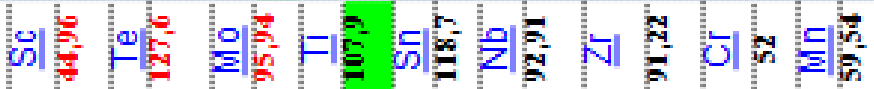

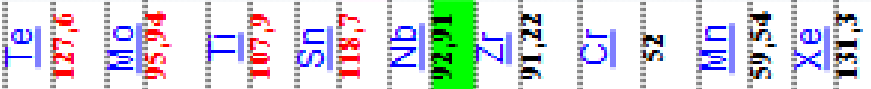

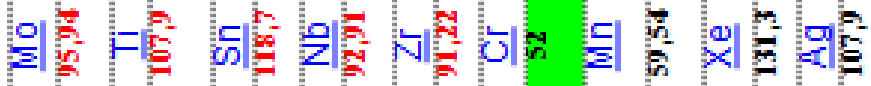

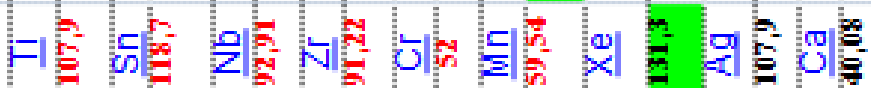

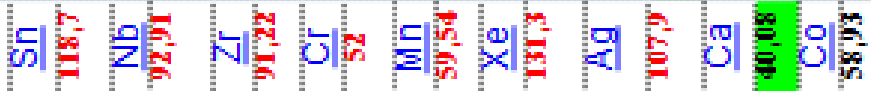

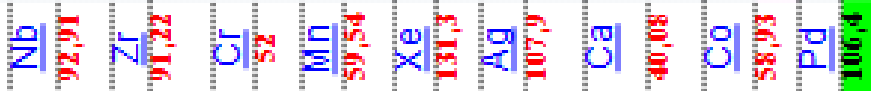




\section{Atomic weight-fragment 3 .}

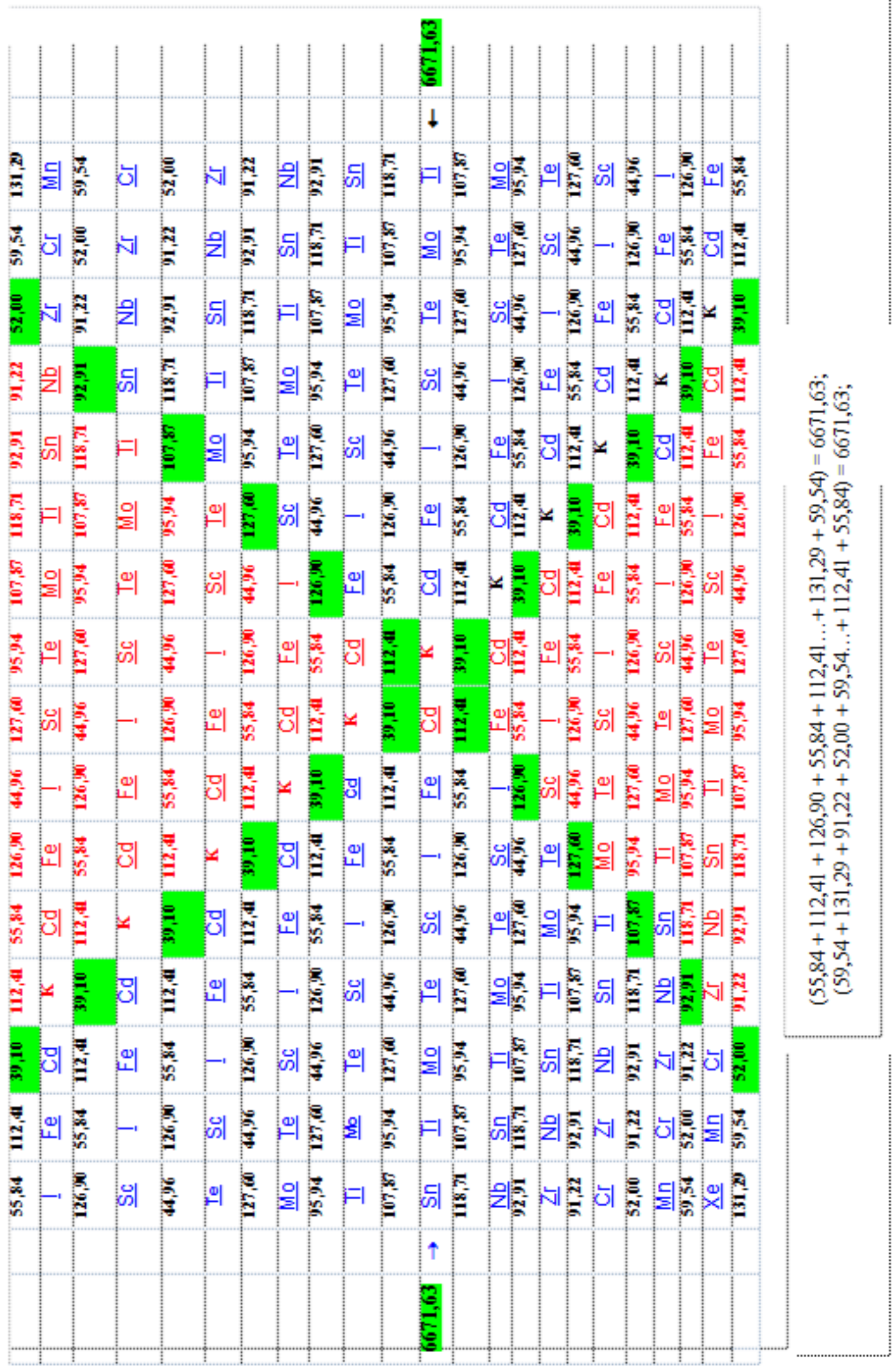


etc.

Table(ListCorrelate( $\{342,1841,57,2754\},\{6671,63\}^{\wedge}\left(\mathrm{x}^{\wedge}\right.$ conjugate $\left.\left.),\{1\}\right)\right)$

Input:

ListCorrelate $\left[\{342,1841,57,2754\},\{6671,63\}^{x^{x^{*}}},\{1\}\right]$

Result:

$$
\begin{aligned}
& \left\{263 \times 7^{x^{x^{*}}+1} 9^{x^{x^{*}}}+34 \times 7^{x^{x^{*}}} 9^{x^{x^{*}}+2}+57 \times 7^{x^{x^{*}}+1} 953^{x^{x^{*}}},\right. \\
& \left.19 \times 3^{2 x^{x^{*}}+1} 7^{x^{x^{*}}}+38 \times 7^{x^{x^{*}}} 9^{x^{x^{*}}+1}+263 \times 7^{x^{x^{*}}+1} 953^{x^{x^{*}}}+2754 \times 6671^{x^{x^{*}}}\right\}
\end{aligned}
$$

Making a sequence of all phenomena in Periodic system Table is conducted according to the exact mathematical laws (for such descriptions we can use theory of systems and cybernetics. The results of our research show that the processes of sequencing the periodic table are conditioned and arranged not only with chemical and biochemical lawfulness, but also with program, cybernetic and informational lawfulness too. Translation of the chemical language of these table into a digital language may be very useful for developing new methods of predicting of phenomenon in chemistry, biochemistry, genetic, medicine and other natural sciences.

\section{CONCLUSIONS}

Indeed, the sequencing of the Periodic system Table is determined not only by chemical features, but also by cybernetic and information principles. For this reason, research in this field deals more with the quantitative rather than qualitative characteristics of chemical information and its chemical basis. For the purposes of this paper, specific physical and chemical factors have been selected in order to express the chemical information for chemical elements. Numerical values are them assigned to these factors, enabling them to be measured. In this way it is possible to determine of a connection really exists between the quantitative ratios in the process of transfer of biochemical information and the qualitative appearance of the chemistry. To select these factors, preference is given to classical physical and chemical parameters, including the atomic numbers in the relevant chemical elements, their analog values, the position in periodic table, and their frequencies. Going through these parameters, it becomes clear that there is a mathematical relationship between quantitative ratios and the qualitative appearance of the chemical elements and that there is a measurement method that can be used to describe the biochemistry of those elements. 


\section{References}

[1] L. Kurić, International Letters of Chemistry, Physics and Astronomy 10 (2014) 62-73.

[2] L. Kurić, J. Comput Sci Biol 2 (2009) 101-116.

[3] L. Kurić, Journal de la Societe de statistique de Paris 127(2) (1986).

[4] L. Kurić, GJMR 10(1) (2010) 15.

[5] L. Kurić, Advances and Applications in Bioinformatics and Chemistry (2010) 45-58.

[6] L. Kurić, GJMR 1(1) (2010) 15.

[7] L. Kurić, International Journal of Computer Technology and Application 2(2) (2011) 216-241.

[8] L. Kurić, International Journal of Computer Technology and Application 2(2) (2011) 258-273.

[9] L. Kurić, Journal of Chemical Engineering and Material Science 2(5) (2011).

[10] L. Kurić, International Letters of Chemistry, Physics and Astronomy 11(3) (2014) 202213.

[11] L. Kurić, International Letters of Chemistry, Physics and Astronomy 12 (2014) 31-50.

[12] L. Kurić, International Letters of Chemistry, Physics and Astronomy 13(1) (2014) 11 20. 